\title{
Reconfigurable Circular-Ring Feed Patch Antenna with Tri-Polarization Diversity
}

\author{
Hui Gu, Lei Ge* and Jihong Zhang \\ Shenzhen University, Shenzhen, China
}

In this paper, a reconfigurable circular-ring feed patch antenna for polarization diversity applications is proposed. The reconfigurability is realized by using only two p-i-n diodes which are inserted into the circular feed ring of the aperture-coupled patch antenna. With the help of the p-i-n diodes, the polarization of the antenna is able to be altered among $45^{\circ}$ linearly polarized (LP), left-hand circularly polarized (LHCP), and right-hand circularly polarized (RHCP) states. A fully-functional prototype is fabricated and measured. The measurement agrees well with the simulation and three polarization states are obtained.

OPEN ACCESS

Edited by: Lei Guo,

The University of Queensland,

Australia

Reviewed by:

MEI LI,

Chongqing University, China

Mohd Fairus Mohd Yusoff,

University Technology Malaysia,

Malaysia

Juhua Liu,

Sun Yat-sen University, China

*Correspondence:

Lei Ge

leige@szu.edu.cn

Specialty section:

This article was submitted to Radiation Detectors and Imaging,

a section of the journal

Frontiers in Physics

Received: 03 August 2021 Accepted: 01 September 2021 Published: 13 September 2021

Citation:

Gu H, Ge L and Zhang J (2021) Reconfigurable Circular-Ring Feed

Patch Antenna with Tri-

Polarization Diversity.

Front. Phys. 9:752505.

doi: 10.3389/fphy.2021.752505
Owing to the merits of tri-polarization diversity, low profile and simple structure, the proposed design is a great candidate for future wireless systems.

Keywords: antenna, patch antenna, reconfigurable antenna, polarization reconfiguration, switch

\section{INTRODUCTION}

With the popularity of wireless communication technologies, reconfigurable antennas with switchable linearly polarized (LP) or circularly polarized (CP) waves have been receiving much attention. They can enhance the performance of radio-frequency systems due to their advantages of reducing polarization mismatch and mitigating multi-path fading effects [1-3]. In the past few years, many works have been undertaken on the design of polarization reconfigurable antennas [4-12]. In [4-7], the designs can provide LP reconfiguration along different polarization directions. CP reconfigurable antennas, with the ability of switching the polarization between left-hand circularly polarized (LHCP) and right-hand circularly polarized (RHCP) states, have been reported in [8-10]. It is observed that these designs can only alter the polarization either between LP modes or between CP modes. In particular, a reconfigurable antenna which can switch its polarization between LP state and CP state is preferred. To realize it, the designs in $[11,12]$ achieved a pair of orthogonal CP waves and an LP wave in a single antenna structure.

Due to the feature of simple structure and easy fabrication, the resonant-type series feeding crossaperture coupled microstrip antenna have been studied over the past decades. This type of antenna is flexible to realize $\mathrm{CP}$ operation with wide axial ratio bandwidth and flat gain bandwidth. Its detailed radiating mechanism has been systematically analyzed based on the theory of the transmission line model [13] and the reciprocity method [14]. Based on this idea, it is possible to design dual-band CP radiation. In [15], the authors employed a series feed line to couple a diamond-shaped slot to a ring and a cross-slot to a patch in series in order to realize a dual-band CP antenna. To minimize its size, several modifications, including the cross-shaped slots replaced by multiple slots and the series feeding line replaced by an open-ended circular ring, were made to obtain a more compact one as explored in [16]. Therefore, the series feeding aperture-coupled microstrip antenna becomes attractive for polarization reconfigurable designs. 


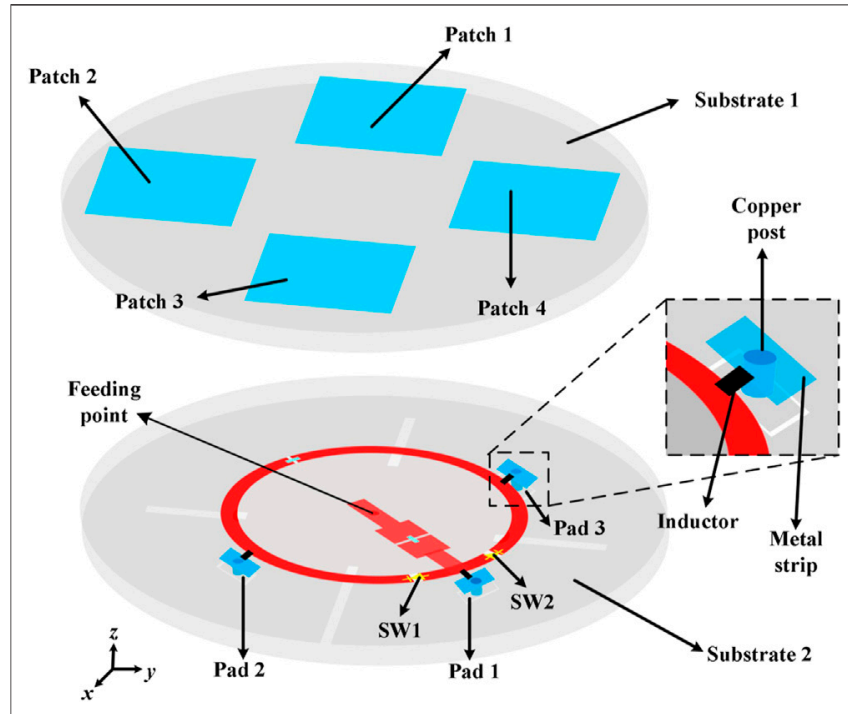

FIGURE 1 | Three-dimensional configuration of the proposed polarization reconfigurable antenna.

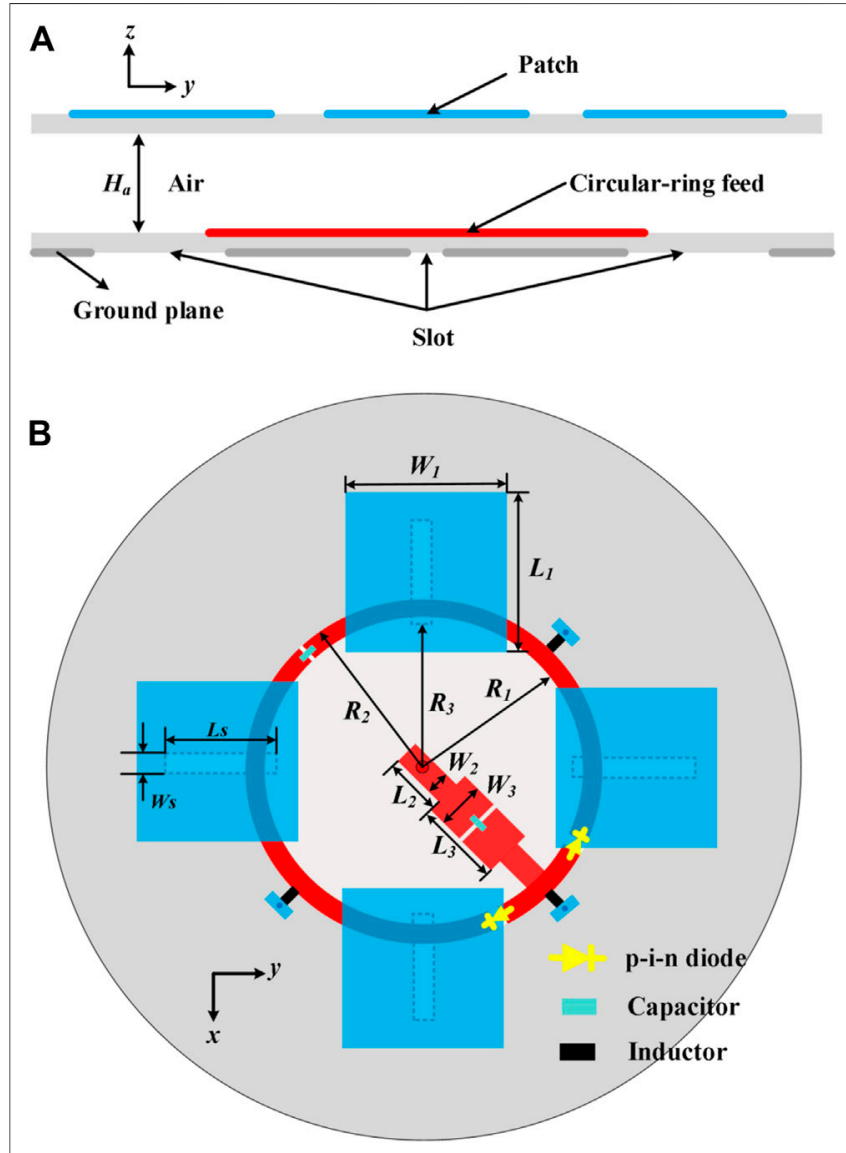

FIGURE 2 | Side and top views of the proposed polarization reconfigurable antenna: (A) Side view; (B) Top view.
TABLE 1 | Dimensions of the proposed antenna.

\begin{tabular}{lccccc}
\hline Parameters & $\mathbf{H}_{\mathbf{a}}$ & $\mathbf{L}_{\mathbf{1}}$ & $\mathbf{L}_{\mathbf{2}}$ & $\mathbf{L}_{\mathbf{3}}$ & $\mathbf{W}_{\mathbf{1}}$ \\
\hline Values/mm & 2 & 23 & 6.8 & 14 & 23 \\
Parameters & $\mathrm{W}_{2}$ & $\mathrm{~W}_{3}$ & $\mathrm{~L}_{\mathrm{s}}$ & $\mathrm{W}_{\mathrm{s}}$ & $\mathrm{R}_{1}$ \\
Values $/ \mathrm{mm}$ & 2 & 3.6 & 20.5 & 2 & 19.7 \\
Parameters & $\mathrm{R}_{2}$ & $\mathrm{R}_{3}$ & - & - & - \\
Values/mm & 21.7 & 19.5 & - & - & -
\end{tabular}

TABLE 2 | Different polarization states.

\begin{tabular}{lll} 
State & sw1 & sw2 \\
\hline RHCP & ON & OFF \\
LHCP & OFF & ON \\
LP & ON & ON
\end{tabular}

In this paper, a polarization reconfigurable circular-ring fed patch antenna is proposed. Two $\mathrm{p}-\mathrm{i}-\mathrm{n}$ diodes are soldered on the circular feed ring. By controlling the states of the $\mathrm{p}-\mathrm{i}-\mathrm{n}$ diodes, the antenna polarization can be switched between a pair of orthogonal CP modes and an LP mode. In addition, two 68$\mathrm{pF}$ capacitors are used to block DC signals. Compared with the designs presented in [13-16], the proposed antenna can also provide an additional LP state. Compared with tri-polarization diversity antennas demonstrated in $[11,12]$, the proposed design has a much lower profile. Details of the simulated and measured results are presented to validate the proposed design.

\section{ANTENNA DESIGN}

\section{Antenna Geometry}

The three-dimensional configuration of the proposed antenna is illustrated in Figure 1, which consists of two microwave FR4 substrates (Substrate 1 and 2) and an air layer. Both the FR4 substrates are with the same thickness of $0.8 \mathrm{~mm}$, radius of $48 \mathrm{~mm}$ and relative permittivity of 4.4 , and they are separated by an air-gapped layer with a height of $H_{a}$. The side and top views are presented in Figure 2, and the detailed dimensions of the proposed antenna are given in Table $\mathbf{1}$. For the radiator, there are four square patches printed on the top surface of Substrate 1 with $90^{\circ}$ rotation between each other. On the top surface of the lower FR4 substrate, there is a switchable circular-ring microstrip line, and the bottom is a ground plane with four identical slots right below the four patches. As such, the proposed antenna can be excited by an aperture-coupling scheme. It should be noted that a conventional aperturecoupled structure is realized by placing a microstrip feedline above the coupling slots. But in this design, the configuration is right reversed. The purpose of this arrangement is for the purpose of directly feeding the antenna with a coaxial probe in the center. 

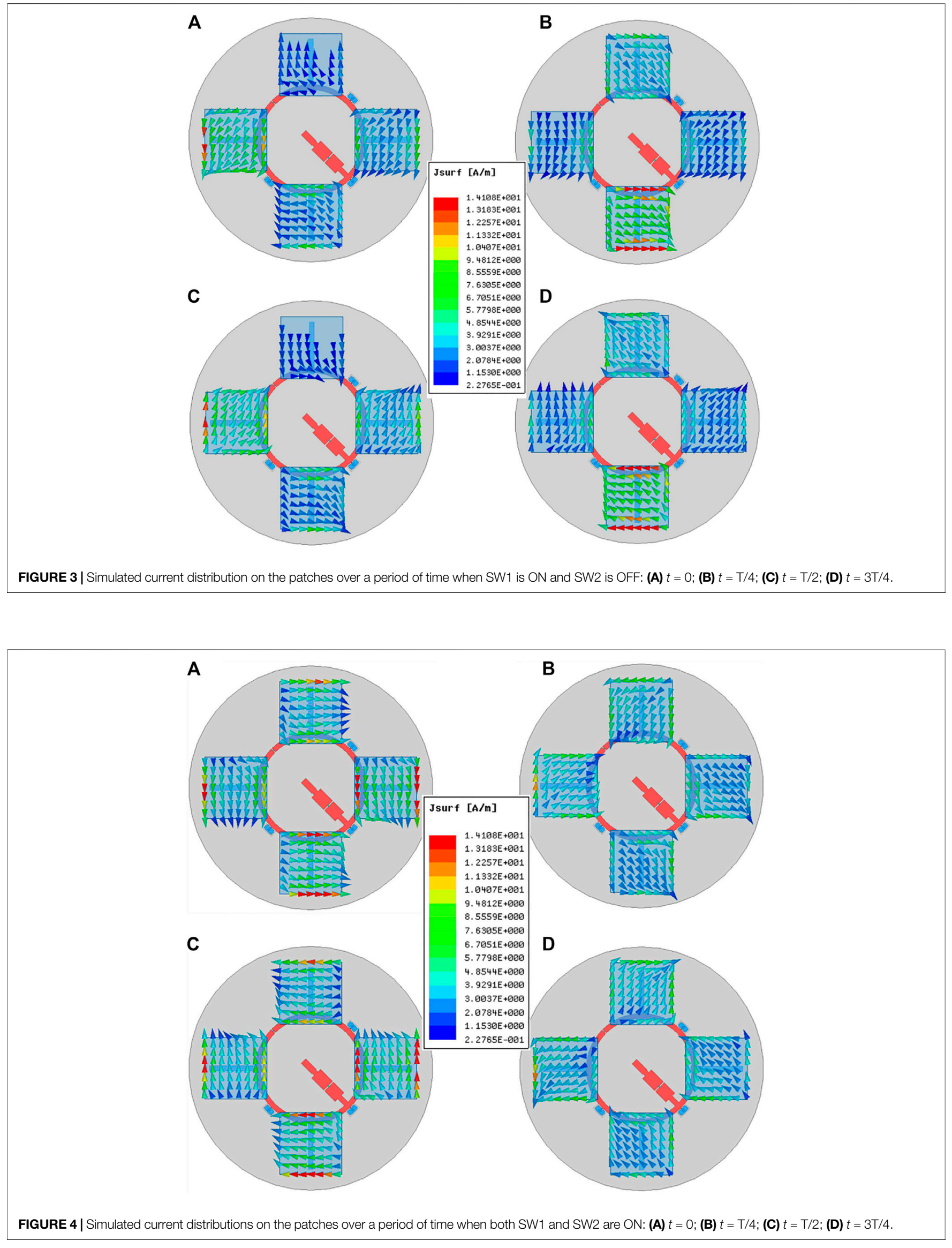


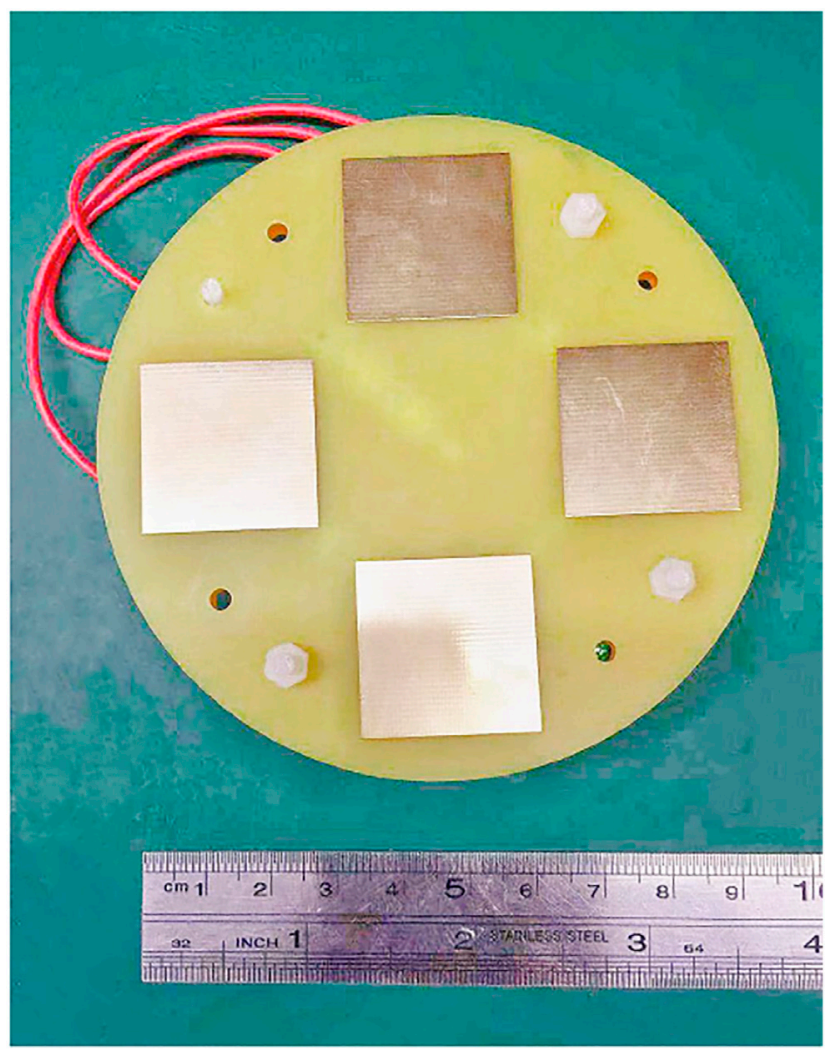

FIGURE 5 | Photograph of the proposed antenna.

\section{Operating Principle}

In the proposed design, two p-i-n diodes, named SW1 and SW2, are inserted into the circular-ring microstrip line, constituting a direction-switchable line. The $\mathrm{p}$-i-n diodes used here as switches, are MADP-000907-14020P from MACOM [17]. The diode is ON when it is forward biased by using a $1.5-\mathrm{V}$ DC voltage. If the diode is unbiased, it will be turned OFF. To control the states of the diodes, three groups of DC pads are used in the design, labeled as Pad 1, Pad 2, and Pad 3. Three slots are etched on the ground plane to ensure the independent control of the DC pads. Besides, two 68-pF capacitors are used to block DC signals and three 47$\mathrm{nH}$ inductors are utilized to isolate DC lines from RF signals. It needs to be mentioned that the biasing circuit is located below the ground plane with the help of metal strips and copper posts, so the effect of the biasing circuit on the antenna performances is limited.

As illustrated above, the design can switch the polarization among two orthogonal CP states and an LP state. The details are depicted in Table 2. When SW1 is ON and SW2 is OFF, four slots etched on the ground plane are excited with a sequential phase in clockwise direction. Because slots are used as the source to feed the patches, RHCP wave is realized. Similarly, when SW1 is OFF and SW2 is ON, four slots etched on the ground plane are excited with a sequential phase in anticlockwise direction. Hence, LHCP wave is obtained. Finally, the LP state is achieved when both SW1 and SW2 are ON.
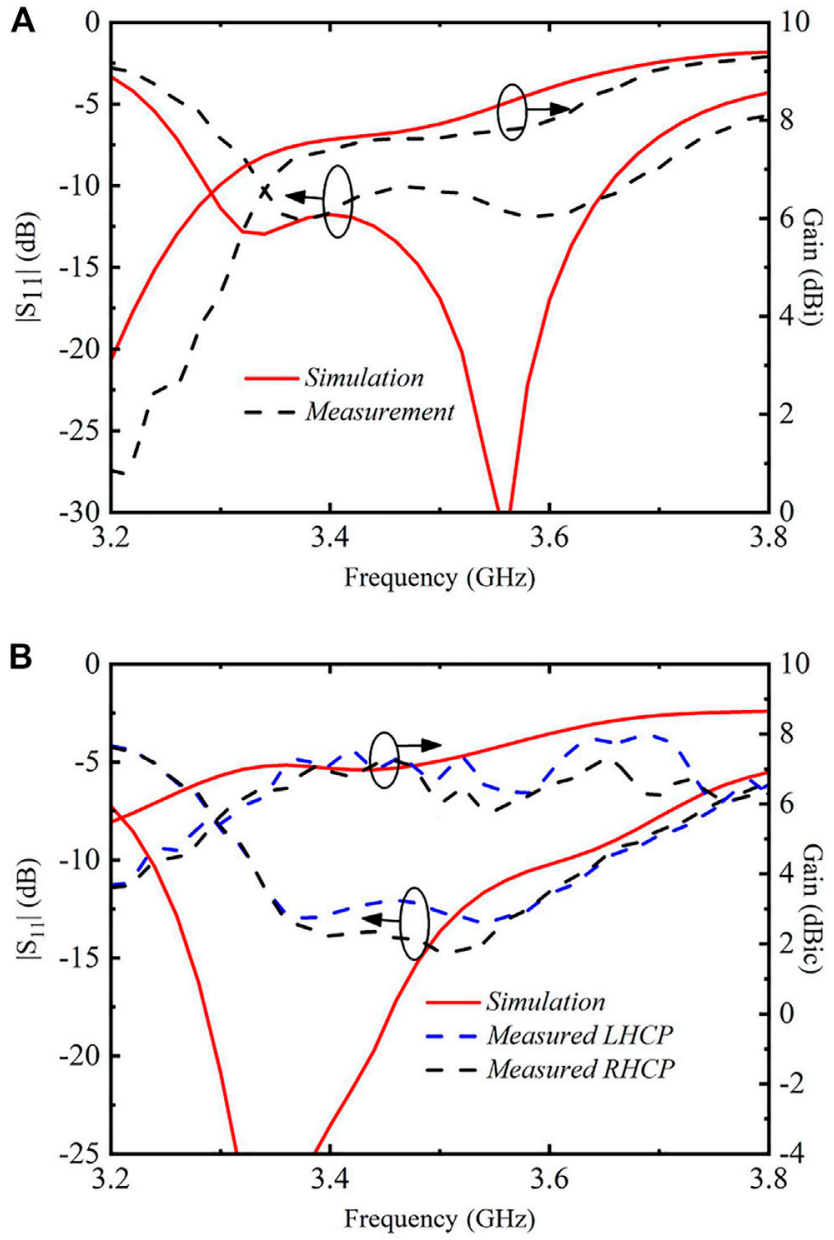

FIGURE 6 | Simulated and measured reflection coefficients and broadside gains of the proposed antenna: (A) LP state; (B) CP states.

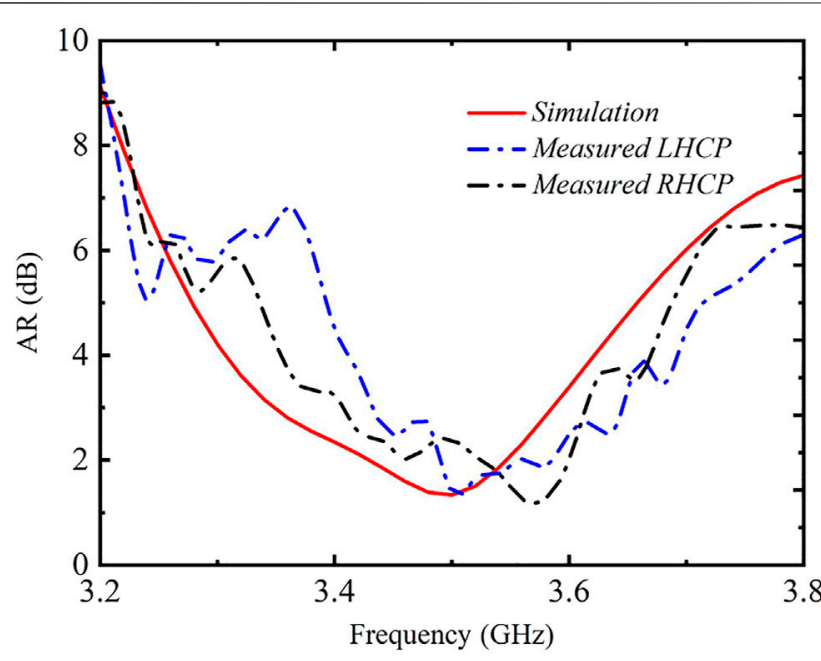

FIGURE 7 | Simulated and measured ARs of the proposed antenna for two CP states. 


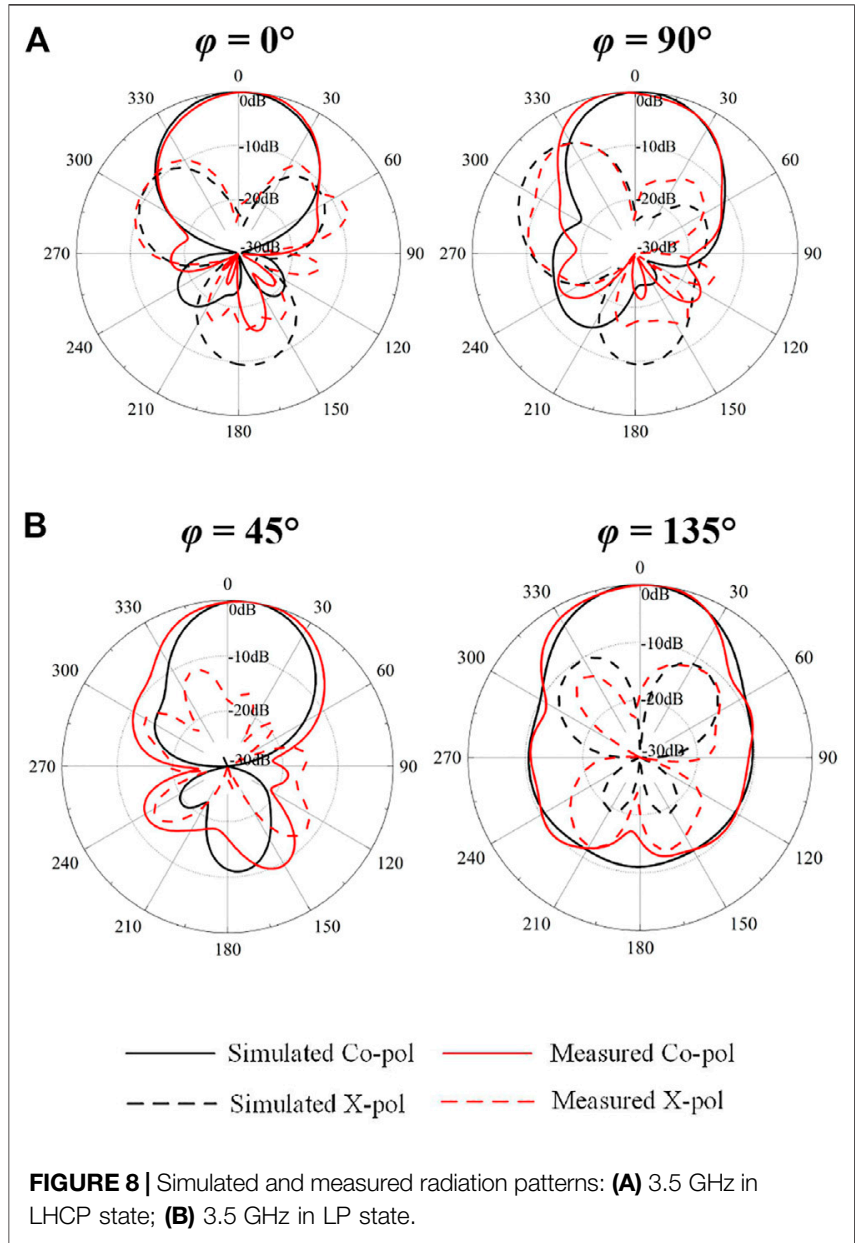

Figure 3 gives the simulated current distribution on the patches over a period when SW1 is ON and SW2 is OFF. At time $t=0$ and $\mathrm{T} / 2$ ( $\mathrm{T}$ is a period of time), the current is along $x$-direction on Patch 1 and Patch 3 and current on the other two patches is minimized. At time $t=\mathrm{T} / 4$ and $3 \mathrm{~T} / 4$, the $y$-direction current is dominant on Patch 2 and Patch 4 and the current on Patch 1 and Patch 3 is weak. Therefore, CP radiation is realized when SW1 is ON and SW2 is OFF. According to the rotated direction of the current, it is demonstrated that the antenna works under the RHCP state. In terms of LP state, the simulated current distribution on the patches over a period when both SW1 and SW2 are $\mathrm{ON}$ is presented in Figure 4. As the figure illustrates, at time $T=0$, current on Patch 2 and Patch 4 flows along the $x$ direction, and current on Patch 1 and Patch 3 flows along the $y$ direction. Thus, the resultant current is along the direction of $\varphi=45^{\circ}$. When $t=\mathrm{T} / 2$, the resultant current is opposite to that of $t=0$. As a result, $45^{\circ} \mathrm{LP}$ radiation is achieved.

\section{SIMULATED AND MEASURED RESULTS}

For experimental validation, a fully-functional prototype was fabricated and measured. Figure 5 dsplays the photograph of the constructed prototype. As seen from the photograph, the two PCB layers are connected with each other using some plastic supports. The Ansys HFSS is used in the simulation [18]. The measured results, including S-parameters, the gain values and radiation patterns were obtained with a Keysight E5080A network analyzer and a near-field antenna measurement system, respectively.

The simulated and measured reflection coefficients of the proposed antenna under different states are given in Figure 6. As Figure 6 shows, the measured $-10 \mathrm{~dB}$ impedance bandwidth is almost the same for two CP states: $9.7 \%$ from 3.32 to $3.66 \mathrm{GHz}$. The measured $-10 \mathrm{~dB}$ impedance bandwidth for LP state is $9.7 \%$ from 3.34 to $3.68 \mathrm{GHz}$. Figure 7 depicts the simulated and measured axial ratios (ARs) for two CP states. The measured AR bandwidth is from 3.44 to $3.64 \mathrm{GHz}$ for LHCP state and from 3.42 to $3.6 \mathrm{GHz}$ for RHCP state. The discrepancy between the simulations and the measurements is mainly due to the fabrication tolerance and the imperfect environment of the measured system. Therefore, the measured effective operation band for both $\mathrm{S}_{11} \leq-10 \mathrm{~dB}$ and $\mathrm{AR} \leq$ $3 \mathrm{~dB}$ is from 3.43 to $3.6 \mathrm{GHz}$.

The simulated and measured broadside gains of the proposed antenna in three states are also depicted in Figure 6. Within the effective bandwidth, for two orthogonal CP states, the measured gain is around $7 \mathrm{dBic}$; for the LP state, the measured gain is from 7.5 to $8.7 \mathrm{dBi}$. The measured efficiencies over the effective band is more than $75 \%$ for all three polarization states.

Figure 8 gives the simulated and measured radiation patterns in LHCP and LP states at 3.5 GHz. Patterns in RHCP states are not given for brevity. From the results, it is seen that unidirectional radiation patterns are obtained. As shown in Figure 8 (a), the measured co-polarized fields at $\theta=90^{\circ}$ are approximately $22 \mathrm{~dB}$ stronger than the corresponding crosspolarized counterpart. Hence, CP radiation is realized. As for LP state, the measured cross-polarization levels are as low as no more than $-18 \mathrm{~dB}$ in both planes. The measured radiation patterns agree well with the simulations in the broadside direction. The differences between simulated and measured radiation patterns in the back side are mainly owing to the influence of DC cables.

TABLE 3 | Comparison between proposed and other reported polarization reconfigurable antennas.

\begin{tabular}{llccr}
\hline Ref & Antenna type & States & Effective BW/\% & Size \\
\hline$[10]$ & Patch & 2 & 20 & $0.7 \lambda_{0} \times 0.7 \lambda_{0} \times 0.09 \lambda_{0}$ \\
{$[11]$} & Patch & 3 & 16 & $0.77 \lambda_{0} \times 0.77 \lambda_{0} \times 0.12 \lambda_{0}$ \\
{$[12]$} & ME dipole & 3 & 16 & $1.0 \lambda_{0} \times 1.0 \lambda_{0} \times 0.27 \lambda_{0}$ \\
This Work & Patch & 3 & 5 & $1.1 \lambda_{0} \times 1.1 \lambda_{0} \times 0.04 \lambda_{0}$
\end{tabular}


Table 3 lists a comparison between our work and other reported polarization reconfigurable antennas. Compared with the reported designs, the proposed antenna has a much lower profile with three different polarization states.

\section{CONCLUSION}

A reconfigurable circular-ring feed patch antenna has been presented. Three polarization states are achieved by controlling the states of two diodes. A prototype was designed, fabricated and measured to verity the design idea. An overlapped bandwidth of $4.8 \%$ is achieved and radiation patterns are stable within the operating band. Due to merits of tri-polarization diversity, low profile and

\section{REFERENCES}

1. Row J-S, and Hou M-J. Design of Polarization Diversity Patch Antenna Based on a Compact Reconfigurable Feeding Network. IEEE Trans Antennas Propagat (2014) 62(10):5349-52. doi:10.1109/tap.2014.2341271

2. Guo YX, Bian L, and Shi XQ. Broadband Circularly Polarized Annular-Ring Microstrip Antenna. IEEE Trans Antennas Propagat (2009) 57(8):2474-7. doi:10.1109/tap.2009.2024584

3. Pan YM, Zheng SY, and Hu BJ. Wideband and Low-Profile Omnidirectional Circularly Polarized Patch Antenna. IEEE Trans Antennas Propagat (2014) 62(8):4347-51. doi:10.1109/tap.2014.2323412

4. Wu F, and Luk K-M. A Reconfigurable Magneto-Electric Dipole Antenna Using Bent Cross-Dipole Feed for Polarization Diversity. Antennas Wirel Propag Lett (2017) 16:412-5. doi:10.1109/lawp.2016.2581259

5. Gu H, Wang J, Ge L, and Sim C-Y -D. A New Quadri-Polarization Reconfigurable Circular Patch Antenna. IEEE Access (2016) 4:4646-51. doi:10.1109/access.2016.2600250

6. Wong H, Lin W, Huitema L, and Arnaud E. Multi-polarization Reconfigurable Antenna for Wireless Biomedical System. IEEE Trans Biomed Circuits Syst (2017) 11(3):652-60. doi:10.1109/tbcas.2016.2636872

7. Lin W, and Wong H. Polarization Reconfigurable Aperture-Fed Patch Antenna and Array. IEEE Access (2016) 4:1510-7. doi:10.1109/ access.2016.2552488

8. Zhang L, Gao S, Luo Q, Young PR, and Li Q. Wideband Loop Antenna with Electronically Switchable Circular Polarization. Antennas Wirel Propag Lett (2017) 16:242-5. doi:10.1109/lawp.2016.2570859

9. Khidre A, Lee K-F, Yang F, and Elsherbeni AZ. Circular Polarization Reconfigurable Wideband E-Shaped Patch Antenna for Wireless Applications. IEEE Trans Antennas Propagat (2013) 61(2):960-4. doi:10.1109/tap.2012.2223436

10. Lin W, and Wong H. Wideband Circular-Polarization Reconfigurable Antenna with L-Shaped Feeding Probes. Antennas Wirel Propag Lett (2017) 16:2114-7. doi:10.1109/lawp.2017.2699289

11. Wang KX, and Wong H. A Reconfigurable CP/LP Antenna with Cross-Probe Feed. Antennas Wirel Propag Lett (2017) 16:669-72. doi:10.1109/ lawp.2016.2598248 simple structure, the proposed antenna is promising for future wireless systems.

\section{DATA AVAILABILITY STATEMENT}

The original contributions presented in the study are included in the article/Supplementary Material, further inquiries can be directed to the corresponding author.

\section{AUTHOR CONTRIBUTIONS}

All authors listed have made a substantial, direct, and intellectual contribution to the work and approved it for publication.

12. Ge L, Yang X, Zhang D, Li M, and Wong H. Polarization-Reconfigurable Magnetoelectric Dipole Antenna for 5G Wi-Fi. Antennas Wirel Propag Lett (2017) 16:1504-7. doi:10.1109/lawp.2016.2647228

13. Aloni E, and Kastner R. Analysis of a Dual Circularly Polarized Microstrip Antenna Fed by Crossed Slots. IEEE Trans Antennas Propagat (1994) 42: 1053-8. doi:10.1109/8.309996

14. Kim H, Lee BM, and Yoon Y-J. A Single-Feeding Circularly Polarized Microstrip Antenna with the Effect of Hybrid Feeding. Antennas Wirel Propag Lett (2003) 2:74-7. doi:10.1109/lawp.2003.813382

15. Chang T-N, and Lin J-M. Serial Aperture-Coupled Dual Band Circularly Polarized Antenna. IEEE Trans Antennas Propagat (2011) 59(6):2419-23. doi:10.1109/tap.2011.2144553

16. Chang TN, and Lin JM. A Novel Circularly Polarized Patch Antenna with a Serial Multislot Type of Loading. IEEE Trans Antennas Propagat (2007) 55(11):3345-8. doi:10.1109/tap.2007.908846

17. MACOM. MADP-000907-14020P PIN Diode (2015). Available: http://www. macom.com/products/product-detail/MADP- 000907-14020P.

18. Ansys Corp. HFSS: High Frequency Structure Simulator Based on the Finite Element Method (2021). Available: http://www.ansys.com.

Conflict of Interest: The authors declare that the research was conducted in the absence of any commercial or financial relationships that could be construed as a potential conflict of interest.

Publisher's Note: All claims expressed in this article are solely those of the authors and do not necessarily represent those of their affiliated organizations, or those of the publisher, the editors and the reviewers. Any product that may be evaluated in this article, or claim that may be made by its manufacturer, is not guaranteed or endorsed by the publisher.

Copyright $\Subset 2021 \mathrm{Gu}, \mathrm{Ge}$ and Zhang. This is an open-access article distributed under the terms of the Creative Commons Attribution License (CC BY). The use, distribution or reproduction in other forums is permitted, provided the original author(s) and the copyright owner(s) are credited and that the original publication in this journal is cited, in accordance with accepted academic practice. No use, distribution or reproduction is permitted which does not comply with these terms. 